\title{
Mortalite ve medikal komplikasyonlar
}

\section{Mortality and medical complications}

\author{
Murat Celal Sözbilen
}

Dr. Behçet Uz Çocuk Hastalıkları ve Cerrahisi Eğitim Araştırma Hastanesi, Ortopedi Kliniği, İzmir

\begin{abstract}
Total diz artroplastisi modern ortopedik cerrahinin en ileri gelişmelerinden biri olup güvenli ve etkili bir prosedürdür. Ancak, diz artroplastisi ölümle dahi sonuçlanabilen birçok ciddi komplikasyon ortaya çıkarabilir. Cerrahi ve anestezi alanındaki son gelişmeler elektif diz artroplastisi sonrası mortalite oranlarını belirgin düzeyde düşürdü. Buna rağmen modern tıp sayesinde ortalama yaşam süresinin uzaması, özellikle kardiyak ve diğer sistemik hastalığı olanların daha iyi sağkalımının olması, primer diz artroplastisinin bu hastalara uygulanma sıklığını arttırmıştır. Dolayısıyla, ortopedistlerin diz artroplastisi sonrası medikal komplikasyonlarla daha yüksek sıklıkta karşılaşması beklenebilir. Mevcut sistemik kronik hastalıkların varlığı komplikasyonların hatta mortalitenin artışıyla doğrudan ilişkilidir. Koroner arter hastalığı, diyabet, hipertansiyon ve benzeri çok çeşitli komorbiditelerle karşılaşılabilmektedir ve bunların ameliyat öncesi hastaya özgün ve multidisipliner olarak değerlendirilmesi gereklidir. Bu amaçla geliştirilen ASA (American Society of Anesthesiologists) ve $\mathrm{CCl}$ (Charlson Comorbidity Index) skorlama sistemleri risk düzeyinin belirlenmesinde kullanılmaktadır. ASA skoru $>3$ ve buna ek olarak ileri yaş $(>80)$, artmış VKi (vücut kitle indeksi) (>40) ve diyabet, cerrahi sonrası komplikasyonların gelişiminde önemli risk faktörleridir. Total diz artroplastisi sonrası mortalite ve hayatı tehdit eden sistemik komplikasyon oranlarının \%2-3 olması, perioperatif dönemde çok özenli bir değerlendirme yapılmasını gerektirir.
\end{abstract}

Anahtar sözcülkler: primer total diz artroplastisi; komplikasyonlar; medikal komplikasyonlar; sistemik komplikasyonlar; mortalite
Total knee arthroplasty represents one of the greatest advances in modern orthopaedic surgery; in addition, it is a safe and effective procedure. However, knee arthroplasty is associated with several serious potential complications and even with death. Recent advances in surgical and anesthesia techniques have contributed to a marked decrease in mortality after total knee arthroplasty. Nevertheless, modern medical care results in longer life expectancy, and provides better survival of patients with cardiac and other systemic illnesses in particular; therefore, the frequency of application of primary knee arthroplasty to these patients increased. Hence, it is possible that orthopaedic surgeons may observe a higher prevalence of medical complications in their patients who have elective knee arthroplasty. Pre-existing systemic diseases and comorbidities have been associated with increased complications and mortality. A wide variety of comorbidities may be encountered, such as coronary artery disease, diabetes, hypertension, and they should be considered patient specific and multidisciplinary before the operation. For this purpose, ASA (American Society of Anesthesiologists) and CCI (Charlson Comorbidity Index) scoring systems are used to determine the risk level. In addition to ASA classification score $(>3)$, patient age $(>80)$, BMI $(>40 \mathrm{~kg} / \mathrm{m} 2)$, and diabetes were identified as risk factors for the development of complications following surgery. The 2-3\% mortality or major complication rate that is found for patients who underwent total knee arthroplasty confirms the need for diligent medical management during the perioperative period.

Key words: primary total knee arthroplasty; complications; medical complications; systemic complications; mortality
M

edikal komplikasyonlar, hastayı sistemik olarak etkileyen, ameliyat sonrası dönemde 30 günlük, 90 günlük ya da uzun dönemde ortaya çıkabilen ve mevcut komorbiditeyi arttırabilen komplikasyonlardır. ${ }^{[1]}$ Total diz protezi (TDP) sonrası en sık karşılaşılanlar cerrahiye bağı lokal komplikasyonlar olsa da, bu sistemik komplikasyonlar hasta hayatını tehdit eden durumlar ortaya çıkarabilir. Bu açıdan, son dönemde minimal invaziv artroplastinin artışı ile birlikte, günübirlik uygulanan cerrahiler sonrası hastanede kalış süreleri azaltılmaktadır. Artan maliyetler, hastane enfeksiyonları, erken rehabilitasyonun evde yapılma isteği gibi nedenlerle tercih edilen bu durum, ciddi sistemik komplikasyonların 24 saat sonrası dönemde hastane dışında ortaya çıkabileceğini göstermiştir. ${ }^{[2]}$ Hayatı tehdit edebilen bu medikal

- İletişim adresi: Dr. Murat Celal Sözbilen, Dr. Behçet Uz Çocuk Hastalıkları ve Cerrahisi Eğitim Araştırma Hastanesi, Ortopedi Kliniği, Konak, İzmir Tel: 0505 - 2997220 e-posta: muratcelal@hotmail.com

- Geliș tarihi: 19 Aralık 2018 Kabul tarihi: 19 Aralık 2018 
komplikasyonların belirlenmesi için geliştirilen ASA (American Society of Anesthesiologists) skor sistemi ve $\mathrm{CCl}$ (Charlson Comorbidity Index) benzeri sınıflamalarla sistemik hastalıklar ve komorbidite için değerlendirme yapılabilse de, yapılan çalışmalar bu komplikasyonların beklenmedik şekilde de ortaya çıkabileceğini göstermektedir. ${ }^{[3]}$ Bu nedenle, hastanede kalış süresini ve hastaya özgü risk faktörlerini belirlemenin önemi artmaktadır. Bunu sağlamak için ise hastaya özgü, multidisipliner bir yaklaşımla değerlendirme yapılmalıdır. Gelişebilecek sistemik komplikasyonları bilmek ve hazırlıklı olmak günümüzde çok sık uygulanan total diz artroplastisi sonrası hayat kurtarıcı olabilir.

\section{MEDIKAL KOMPLIKASYONLAR}

TDP, olumlu sonuçlarının yanı sıra, bununla ilişkili gelişebilecek ciddi komplikasyonlar ile sağıı bakım masraflarını, engelliliği ve mortalite oranlarını arttırabilmektedir. ${ }^{[4]}$

Son on yılda, diyabet oranı $\% 32$ oranında artarken obezite (vücut kitle indeksi $\geq 30$ ) ise $\% 129$ oranlarında artış gösterdi. ${ }^{[5,6]}$ Obezite dizde osteoartrit gelişiminde majör risk faktörüdür ${ }^{[7]}$; bu bağlamda, günümüzde total diz protezi uygulanan obez hasta sayısı artış göstermektedir. Bunun yanı sıra, genel popülasyonun yaşam süresinin uzaması ve kronik medikal hastalıkların artışı, TDP olacak hastaların sistemik medikal komplikasyonlarla karşılaşma sıklığını arttırmıştır. ${ }^{[4]}$

Sistemik medikal komplikasyonların sıklığının artışı, minimal invaziv cerrahi ve hastanede kalış sürelerinin 24 saate kadar indirilmesiyle önem kazanan mortalite ve morbidite, son yıllarda yayımlanmış geniş olgu serilerini içeren ayrıntılı çalışmalar ile değerlendirilmiştir. Bunların bir kısmı tek merkez bölgesel çalışmalar iken $^{[2,8]}$, bir kısmı da ulusların kayıt sistemlerine dayanan geniş serilerdir. ${ }^{[9,10]}$

$\mathrm{Bu}$ geniş olgu serileri içeren çalışmalarda da olmak üzere, hastaların medikal komplikasyonları sistemik ve lokal komplikasyonlar olarak iki ana grupta değerlendirilmiştir. Sistemik medikal komplikasyonlar da majör ve minör olarak sınıflandırılmıştır (Tablo 1). ${ }^{[2,8-10]}$ Komplikasyonlar kompleks medikal girişim gerektiriyor, hayatı tehdit ediyor ya da fonksiyonel bozulma gösteriyorsa, majör komplikasyon olarak değerlendirilir. Minör komplikasyonlar ise gözlem gerektiren veya ek medikal tedavi gerektiren durumlardır. ${ }^{[2,8,9,11]}$

\section{KOMPLIKASYONLARIN INSIDANSI}

Yapılan bazı geniş seri çalışmalarda, majör komplikasyon insidansının \%4,4 ile \%7,7 arasında değişiklik gösterdiği görüldü.. ${ }^{[2,8,12]}$ Higuera ve ark. (2011), 65 yaş üstü hastalarda (ort. yaş $74 \pm 6,3$ ) yaptıkları ileriye dönük kohort çalışmada \%11 oranında majör komplikasyon oranı tespit etmişlerdir. ${ }^{[11]}$ Bunun yanı sıra, yaşın sistemik komplikasyon açısından hastalarda önemli bir prediktör oluşturduğunu belirtmişlerdir. Bu ilişki birçok diğer çalışmada da gösterilmiştir. ${ }^{[2,8,13,14]}$ Belmont ve ark. (2014), geriye dönük olarak ulusal verilerden değerlendirdikleri 15321 hastanın 280'inde $(\% 1,83)$ majör sistemik komplikasyon, 491 (\%3,21) hastada ise minör sistemik komplikasyon görülmüştür (Tablo 2). ${ }^{[10]}$

Aynı çalışmada değerlendirilen hastaların majör sistemik komplikasyonlarının \%46,6'sı kardiyovasküler kaynaklı iken bunlar içinde en sık görülenin pulmoner emboli olduğu görülmüştür. İdrar yolu enfeksiyonu $(\% 1,49)$ ve derin ven trombozu $(\% 1,34)$ en sık görülen minör sistemik komplikasyonlardır. Higuera ve ark. (2011), ileriye dönük değerlendirdikleri geriatrik hastalarda ( $\geq 65$ yaş) majör komplikasyonlardan en sık kardiyovasküler sorunlar (\%9) görülürken, akut böbrek

Tablo 1. Sistemik komplikasyonlar

\begin{tabular}{ll}
\hline $\begin{array}{l}\text { Major sistemik } \\
\text { komplikasyonlar }\end{array}$ & $\begin{array}{c}\text { Minor sistemik } \\
\text { komplikasyonlar }\end{array}$ \\
\hline Kardiyovasküler & Kardiyovasküler \\
Konjestif kalp yetmezliği & Anjina \\
Koroner arter hastalığı & Sinüs taşikardisi \\
Pulmoner emboli & Hipotansiyon \\
Atriyal fibrilasyon & Nörolojik \\
Miyokard enfarktüs & Transient iskemik atak \\
Pulmoner ödem & Konfüzyon \\
Aritmi & Deliryum \\
Derin ven trombozu & Pulmoner \\
Bradikardi & Atelektazi \\
Hipotansif kriz & Pnömoni \\
Kardiyopulmoner arrest & Gastrointestinal \\
Nörolojik & Ileus \\
Serebrovasküler olay & Clostridium difficile enfeksiyonu \\
Anoksik beyin hasarı & Üriner sistem \\
Nöbet & Idrar yolları enfeksiyonu \\
Pulmoner & Üriner retansiyon \\
Solunum yetmezliği & Genel \\
Aspirasyon pnömonisi & Anemi \\
Pnömotoraks & Elektrolit imbalansı \\
Gastrointestinal & \\
Gastrointestinal kanama & \\
Obstrüksiyon & \\
Toksik megakolon & \\
Genel & \\
Akut böbrek yetmezliği & \\
Sepsis & \\
Bakteriyemi & \\
Fungemi & \\
Multi-organ yetmezliği & \\
& \\
& \\
& \\
&
\end{tabular}


Tablo 2. Primer TDP sonrası komplikasyon ve mortalite insidansları

\begin{tabular}{|c|c|c|c|c|c|c|}
\hline Çalışma & $\begin{array}{l}\text { Takip süresi } \\
\text { (gün) }\end{array}$ & $\begin{array}{l}\text { Hasta sayısı } \\
\text { (n) }\end{array}$ & $\begin{array}{c}\text { Ortalama yaş } \\
(y \mathrm{ll})\end{array}$ & $\begin{array}{c}\text { Tüm komplikasyonlar } \\
(\%)\end{array}$ & $\begin{array}{c}\text { Majör sistemik } \\
(\%)\end{array}$ & $\begin{array}{c}\text { Mortalite } \\
(\%)\end{array}$ \\
\hline Parvizi $(2007)^{[2]}$ & 90 & 670 & 64,5 & 32 & 6,7 & 0,14 \\
\hline Pulido $(2008)^{[8]}$ & 3,8 & 5.173 & 65 & 8,3 & 4,91 & 0,12 \\
\hline Higuera 2010 $)^{[11]}$ & 90 & 304 & 72 & 63,9 & 10,8 & 1 \\
\hline Soohoo $(2006)^{[27]}$ & 90 & 222.684 & 69 & $\mathrm{~N} / \mathrm{A}$ & $0,4 \mathrm{PE}$ & 0,53 \\
\hline Belmont $(2014)^{[10]}$ & 30 & 15.321 & 67,3 & 5,55 & 1,83 & 0,18 \\
\hline Hart $(2015)^{[28]}$ & 30 & 33.816 & 67 & $\mathrm{~N} / \mathrm{A}$ & 2,7 & 0,17 \\
\hline
\end{tabular}

$\mathrm{PE}$, pulmoner emboli; N/A, yok.

yetmezliği $(\% 1,8)$ ikinci sırada yer almıştır. Cerrahi sonrası anemi dışında (\%34) diğer minör komplikasyonlar değerlendirildiğinde; derin ven trombozu (\%6), idrar yolu enfeksiyonu $(\% 5,2)$ ve mental durum sorunlarının (\%5) daha sık oranlarda görüldüğü tespit edilmiştir. Geriatrik popülasyonda genel popülasyona göre göze çarpan, komplikasyon farkının daha fazla oranda olması dışında, mental durum değişikliklerinin bu popülasyonda daha fazla görülmesi olmuştur. Geriatrik yaş grubunda (>65 yaş) yaşın, anestezi tipinin, koroner arter hastalığı ve kalp yetmezliği gibi bazı komorbid durumların komplikasyon oranını anlamlı düzeyde arttırdığı tespit edilmiştir. ${ }^{[11]}$

Pulido ve ark.'nın (2008) 15.323 total eklem replasmanının incelendiği çalışmalarında, $955(\% 7,1)$ hastada sistemik komplikasyon görülürken kalan 469 $(\% 3,47)$ hastada minör komplikasyon gelişmiştir. En sık komplikasyonun primer ve revizyon diz protezinde görüldüğü (\%62) tespit edilmiştir. En çok hayatı tehdit eden komplikasyonlar kardiyovasküler kaynaklı (\%74) iken, diğer çalışmalarda olduğu gibi en sık pulmoner emboliyle karşılaşılmıştır (tümünün \%1,12'si). Sistemik 469 minör komplikasyonun beş sistemi de etkilediği görülmüş olup, bunlar çok farklı kategorilerde yer almışlardır (Tablo 2). ${ }^{[8]}$

\section{RISK FAKTÖRLERi}

Yapılan geniş serili çalışmalarda, majör sistemik komplikasyonların insidansını potansiyel olarak etkileyebilecek faktörler değerlendirildiğinde, bazı faktörlerin anlamlı düzeyde ortak olduğu görülmüştür. Illeri yaş, bu bağlamda en anlamlı düzeyde komplikasyonlara neden olan belirleyici (prediktör) olarak tespit edilmiştir; diğer risk faktörlerinden bağımsız olarak hem majör hem de minör sistemik komplikasyon oranlarını arttırmaktadır. Her dekad artışında, komplikasyonların \%40 oranında arttığı tespit edilmiştir. Yaş aralığı
75-85 olan grupta, 65-75 yaş grubuna göre herhangi bir komplikasyon açısından rölatif riskin \%43 arttığı görülmüştür. ${ }^{[11]}$ Sadece majör sistemik komplikasyonlar karşılaştırıldığında bile, 60 yaş altı hastalarla 80 yaş üstü hastalar arasındaki riskin büyük oranda yükseldiği görülmüştür (Odds ratio $(95 \% \mathrm{Cl}): 2,77$ [1,86$4,10]) \cdot{ }^{[10]}$ Cinsiyetin ise önemli ölçüde etkili olmadığı belirtilmiştir. ${ }^{[10,11]}$ Hastaya bağlı faktörlerin değerlendirilmesinde $\mathrm{ASA}$ ve $\mathrm{CCl}$ skorlama sistemlerinin sistemik komplikasyonları belirlemede anlamlı ölçüde belirleyici (prediktör) olduğu görülmüştür. ${ }^{[15,16]}$

VKi (vücut kitle indeksi) günümüzde birçok toplumda giderek artan büyük bir sorun ve kronik hastalık kaynağıdır (özellikle diyabet). Ülkemizde de artan oranda olsa da, özellikle ABD'de obezite oranının $\left(\mathrm{VKi}>30 \mathrm{~kg} / \mathrm{m}^{2}\right) \% 34,3$ olduğu, morbid obezitenin ise $\% 428$ oranda artarak \%6'ya eriştiği görülmüştür. Bu ülke veri tabanları kaynaklı yapılan çalışmada 15.321 unilateral primer diz artroplastisi hastasının \%61,2'sinin obez (VKi $>30 \mathrm{~kg} / \mathrm{m}^{2}$ ) ve \%18,2'sinin diyabet olduğu tespit edilmiştir. VKi $>40 \mathrm{~kg} / \mathrm{m}^{2}$ olan hastaların VKi $<25 \mathrm{~kg} / \mathrm{m}^{2}$ hastalara göre herhangi bir komplikasyon riskinin anlamlı düzeyde arttığı (Odds ratio $(95 \% \mathrm{Cl})$ : $1,47[1,09-1,98])$ ve VKi'nin bağımsız risk faktörü olduğu görülmüştür. ${ }^{[10]}$

Sistemik komorbiditelerde en önemli risk faktörünün kardiyak hastalıklar olduğu, özellikle konjestif kalp yetmezliği ve koroner arter hastalığında majör sistemik komplikasyon riskinin genel popülasyona göre 2,643,15 kat, diyabette ise riskin 1,36 kat fazla olduğu görülmüştür. ${ }^{[11]}$

Cerrahiye bağı faktörler değerlendirildiğinde; eş zamanlı bilateral prosedür, revizyon artroplastisi ve uzun operasyon süresinin (>135 dk) hem majör hem minör komplikasyonlar için riski arttırdığı belirlenmiştir. ${ }^{[8,11]}$ Spinal anestezinin ise riski diğer anestezilere göre \%35 azalttığı belirtilmiştir. ${ }^{[11]}$ Başka bir çalışmada ise genel 
anestezinin tüm komplikasyonlar oranını anlamlı şekilde arttırdığı görülmüştür (Odds ratio $(95 \% \mathrm{Cl}): 1,123$ $[1,004-1,269])$. Bu riskin $70-79$ yaş arası hastalarda 50-59 yaş arası referans alındığında arttığı (Odds ratio $(95 \% \mathrm{Cl}): 1,531[1,263-1,856])$ ve 80 yaş üstü hastalarda ise daha da fazla arttığı (Odds ratio $(95 \% \mathrm{Cl})$ : $2,173[1,725-2,737])$ görülmüştür. ${ }^{[17]}$

\section{KOMPLIKASYONLARIN ZAMANLAMASI}

Komplikasyonların zamanlaması cerrahinin tipine göre değişiklik göstermektedir. Farklı çalışmalarda, genellikle elektif kalça ve diz artroplastisiyle birlikte değerlendirildiğinde, hastanın yaşı ve komorbiditesi, komplikasyonların görülme zamanını en çok belirleyen etmen olmuştur. ${ }^{[10,11]}$ Pulida (2008) ve Parvizi (2007) yaptıkları çalışmalarda, komplikasyonların ortaya çıkış süresini ortalama dört gün olarak tespit etmişlerdir. ${ }^{[2,8]}$ Ortalama hastanede yatış süresi ise sırasıyla 3,8 ve 3,9 gün olarak tespit edilmiştir. Genel olarak, sistemik komplikasyonların ilk iki haftada ortaya çıktığı görülmektedir. ${ }^{[10,11]}$

\section{HASTANEDE KALIŞ SÜRESi}

Kronik kalp yetmezliği, koroner arter hastalığı, kronik obstrüktif akciğer hastalığı ve serebrovasküler hastalık durumlarında hastanede kalış sürelerinin uzadığı belirlenmiştir. ${ }^{[2,8,10,11]}$ Özellikle koroner arter hastalığı olanlarda majör komplikasyonların ve re-operasyonun 90 gün içinde anlamlı oranda arttığı görülmüş, buna da çok sayıda transfüzyon ihtiyacının neden olduğu düşünülmüştür. Transfüzyon artışının eklem enfeksiyon riskini, buna bağlı olarak majör komplikasyon ve re-operasyon riskini de arttırdığı düşünülebilir. ${ }^{[18,19]}$

\section{GÜNÜBIRLIK TDP UYGULAMASI VE MEDIKAL KOMPLIKASYONLAR}

Son yıllarda, minimal invaziv artroplasti cerrahileri oldukça popüler olmuştur. ${ }^{[20]}$ Minimal invaziv cerrahinin tercihindeki ana etmenler; daha az kan kaybı, cerrahi sonrası daha az ağrı, hızı ıyileşme, kozmetik açıdan daha iyi bir insizyon skarı ve en önemlisi daha az hastanede kalış süresidir. ${ }^{[2,20,21]}$ Artan maliyetler nedeniyle hastanede kalış süresinin kısaltılmaya çalışılması (cerrahi sonrası 24 saat içinde taburcu) bazı önemli tehlikeleri de beraberinde getirebilmektedir. Minimal invaziv artroplasti cerrahisinde, yumuşak doku hasarı ve insizyon skarı dışında, standart yaklaşımda da uygulanan aynı enstrümantasyon tekniği kullanılmaktadır. Bu nedenle, uygulanan cerrahi standart yaklaşımdaki kadar ciddi bir işlemdir; medüller kanal oyularak yapılmaktadır; bu da, standart yaklaşım kadar potansiyel yağ ve pulmoner emboli riski taşımaktadır. ${ }^{[22]}$ Bunun yanı sıra; aynı anestezi verilmekte, kemik kesileri ve enstrümantasyon da konvansiyonel eklem artroplasti ile aynı uygulanmaktadır.

Parvizi ve ark. (2007), yaptıkları çalışmada bu duruma dikkat çekmiş ve standart artroplastide karşılaşılan ciddi sistemik medikal komplikasyonların minimal invaziv eklem replasmanında da görülebileceğini belirtmişlerdir. Bu durum, şu kritik ve önemli uyarıyı yapmalarına neden olmuştur: Günübirlik cerrahi ya da kısa hastanede kalış süresi sonrası taburcu edilen hastalar, bu hayatı tehdit eden sistemik komplikasyonlarla hastane dışında karşılaşırlarsa nasıl müdahale edilebilecek? ${ }^{[2]}$ Kolisek ve ark.'nın (2007) yaptıkları ileriye dönük randomize çalışmada, minimal invaziv cerrahi ve standart cerrahi arasında medikal komplikasyonlar açısından fark bulunmamış olup hastanede kalış süreleri de yaklaşık olarak aynı tespit edilmiştir (sırasıyla; 4 gün ve 4,1 gün). ${ }^{[23]}$ Tersine, Berger ve ark. (2005) minimal invaziv total diz artroplastisi uyguladıkları ardışık 50 hastanın 48'ini aynı gün öğleden sonra taburcu etmiş, komplikasyonlarda ve tekrar hastaneye başvuru sayısında artış olmadığını belirtmişlerdir. Ancak, çalışmaya dahil etme kriterlerini çok sınırlı tutmuşlar, olabilecek majör sistemik komplikasyon riski taşıyan hastaları dahil etmemişlerdir ( $>80$ yaş, kardiyak hastalık öyküsü vb.).. ${ }^{[2]}$ Gandhi ve ark. (2011) yaptıkları meta-analiz çalışmasında, minimal invaziv cerrahi ile uygulanan TDP'lerin sonuçlarının standart yaklaşımla uygulananlara göre daha iyi olmadığını, hatta komplikasyon oranının daha yüksek olduğunu belirtmişlerdir. Bu bulgular neticesinde, minimal invaziv cerrahinin hastanın kliniğine yansıyabilecek olumsuz durumlar için gereksiz bir risk almak olacağına değinmişlerdir. ${ }^{[25]}$ Bu konudaki tartışma, ilerleyen zaman içerisinde geniş olgu serilerinin sonuçlarına ihtiyaç duymaktadır. Ancak, hayatı tehdit edecek medikal komplikasyonların ortalama dört gün içerisinde geliş̧tiği düşünüldüğünde, kısa yatış sürelerine dikkat edilmelidir. ${ }^{[2,26]}$

\section{MEDIKKAL KOMPLIKASYONLARIN ÖNLENMESi}

Birçok farklı etiyolojiye bağlı olabilen ve hayatı tehdit edecek düzeyden minör sistemik kliniğe kadar geniş bir yelpazede olan medikal komplikasyonlar çok çeşitlilik gösterir. Bu çeşitlilik komplikasyonların öngörülebilirliğini ve önlenmesini güçleştirmektedir. Bu nedenle, cerrahi öncesi bakı ve değerlendirme çok önemlidir. Hastaya özel ve ayrıntılı multidisipliner bir değerlendirme gereklidir. Ancak, bundan önce cerrahi endikasyon doğru hastaya, doğru zamanda konmalıdır. İleri yaş (>80 
yaş), eski kardiyak hastalık öyküsü ya da yüksek risk (koroner arter ve kalp yetmezliği), diyabet, aynı zamanda bilateral cerrahi gibi majör sistemik komplikasyon riskini arttıran faktörler, özellikle cerrahi öncesi değerlendirmede dikkate alınmalıdır.[2,10,11] Multidisipliner ve hastaya özel değerlendirme, ilk planda ortopedi hekimi tarafindan endikasyon kararından sonra alınır. Riskli görülen hastaların dahiliye (kardiyoloji) ve anestezi hekimlerince konsülte edilmesi sağlanmalıdır. Ortopedistlerin bu komorbiditeleri değerlendirmesi, standardize edip kolaylaştırması amacıyla bazı skorlama sistemleri geliştirilmiştir. ASA sınıflaması ve $\mathrm{CCl}$ skorlaması en sık kullanılan değerlendirme araçlarındandır. ASA skorlama sistemi, cerrahi sırasında mortalite ve cerrahi sonrası morbiditede geçerliliği kabul edilmiş bir sistemdir. ${ }^{[15,16]} \mathrm{CCl}$ skorlamasında her indeks skor için riskin; majör komplikasyonlar için \%44, minör komplikasyonlar için ise \%18 arttığı tespit edilmiş, tüm komplikasyonlarla da korele olduğu belirlenmiştir. ${ }^{[11]}$ ASA skorları 3'ün üzerinde olan hastalar için majör komplikasyonların \%58 arttığı, minör komplikasyonların ise \%49 arttığı görülmüştür. ${ }^{[10]}$ Bu skorlama sistemleri kullanılarak, öngörülebilen komplikasyonlar için gerekli konsültasyonlar istenmeli, sonrasında oluşabilecek yoğun bakım gerekliliği için önlemler alınmalıdır.

\section{MORTALITE}

Geniş hasta serilerinin değerlendirildiği çalışmalarda mortalite oranının \%0,12 ile \%1 arasında değiştiği görülmüştür (Tablo 2). Hayatı tehdit eden majör sistemik komplikasyonlar değerlendirildiğinde, en sık mortalite nedeninin kardiyak kaynaklı komplikasyonlar olduğu görülmektedir. Kronik kalp yetmezliği ve koroner arter hastalığı bunların başında yer almaktadır. ${ }^{[2,8,10,11,27,28]}$ Higuera ve ark. (2011), ileriye dönük olarak değerlendirdikleri 302 primer total diz protezi hastasında mortalite oranını $\% 1$ tespit etmişler ve bunları kardiyak nedenlere bağlamışlardır. Mortaliteyi direkt belirleyen risklerin; ileri yaş, $\mathrm{CCl}$ indeks, kronik kalp yetmezliği ve koroner arter hastalığı olduğunu bildirmişlerdir. ${ }^{[11]}$ Belmont (2014) da, ileri yaş ve diyabete ek olarak, ASA skoru $>3$ olan hastaların mortalite riskinin 2,8 kat fazla olduğuna dikkat çekmiştir. ${ }^{[10]} \mathrm{Bu}$ da cerrahi öncesi değerlendirmede $\mathrm{CCl}$ ve ASA skorlama sistemlerinin önemini ortaya koymaktadır.

Bunların yanı sıra, bilateral eş zamanlı total diz protezi uygulamasının yüksek mortalite oranlarıyla ilişkili olduğu görülmüş, ileri yaş ve diyabetin mortalite için bağımsız belirleyici (prediktör) olduğu görülmüştür. ${ }^{[11,29,30]}$
Mortalite sonrası değerlendirmede, bunlara neden olan ana risk faktörü dışında birçok majör sistemik komplikasyonun da eklenerek mortaliteye sebep olduğu bilinmektedir. Neticede, gelişen kardiyak arrest, sistemik şok ve renal yetmezlik mortaliteye sebep olan en özgül komplikasyonlardır.

\section{SONUÇ}

Genel olarak; yaş (>80), cerrahi tipi (bilateral) ve süresi (>135 dk), VKi (>40 kg/m²), anestezi tipi (genel), diyabet ve kardiyak komorbiditeler (özellikle koroner arter hastalığı ve kronik kalp yetmezliği) komplikasyonların ve beklenmeyen sonuçların tahmininde ana rollere sahiptir. Hastaya özel multidisipliner yaklaşım ve risk belirleme sistemlerinin (ASA, $\mathrm{CCI}$ ) kullanılması hayatı tehdit eden komplikasyonlar ve mortalitenin önlenebilmesinde oldukça önemlidir.

\section{KAYNAKLAR}

1. Saucedo JM, Marecek GS, Wanke TR, Lee J, Stulberg SD, Puri L. Understanding readmission after primary total hip and knee arthroplasty: who's at risk? J Arthroplasty 2014;29(2):25660. Crossref

2. Parvizi J, Mui A, Purtill JJ, Sharkey PF, Hozack WJ, Rothman $\mathrm{RH}$. Total joint arthroplasty: when do fatal or near-fatal complications occur? J Bone Joint Surg 2007;89(1):27-32. Crossref

3. Courtney PM, Rozell JC, Melnic CM, Lee GC. Who should not undergo short stay hip and knee arthroplasty? Risk factors associated with major medical complications following primary total joint arthroplasty. J Arthroplasty 2015;30(9):14. Crossref

4. Memtsoudis SG, Della Valle AG, Besculides MC, Gaber L, Laskin R. Trends in demographics, comorbidity profiles, in-hospital complications and mortality associated with primary knee arthroplasty. J Arthroplasty 2009;24(4):51827. Crossref

5. Center for Disease Control and Prevention. 2011 National Diabetes Fact Sheet. Available at: https://www.cdc.gov/ diabetes/pubs/pdf/ndfs_2011.pdf Accessed: December 26, 2018.

6. Ogden $\mathrm{CL}$, Carroll MD. Prevalence of overweight, obesity, and extreme obesity among adults: United States, trends 1960-1962 through 2007-2008. National Center of Health Statistics Health E-Stats, 2010. Available at: https://www. cdc.gov/nchs/data/hestat/obesity_adult_07_08/obesity_ adult_07_08.pdf Accessed: December 26, 2018.

7. Felson DT, Lawrence RC, Dieppe PA, Hirsch R, Helmick CG, Jordan JM, Kington RS, Lane NE, Nevitt MC, Zhang Y, Sowers M, McAlindon T, Spector TD, Poole AR, Yanovski SZ, Ateshian G, Sharma L, Buckwalter JA, Brandt KD, Fries JF. Osteoarthritis: new insights. Part 1: the disease and its risk factors. Ann Intern Med 2000;133(8):635-46.

8. Pulido L, Parvizi J, Macgibeny M, Sharkey PF, Purtill JJ, Rothman RH, Hozack WJ. In hospital complications after total joint arthroplasty. J Arthroplasty 2008;23(6):139-45. Crossref

9. Jain NB, Guller U, Pietrobon R, Bond TK, Higgins LD. Comorbidities increase complication rates in patients having arthroplasty. Clin Orthop Relat Res 2005;(435):232-8. Crossref 
10. Belmont Jr PJ, Goodman GP, Waterman BR, Bader JO, Schoenfeld AJ. Thirty-day postoperative complications and mortality following total knee arthroplasty: incidence and risk factors among a national sample of 15, 321 patients. J Bone Joint Surg Am 2014;96(1):20-6. Crossref

11. Higuera CA, Elsharkawy K, Klika AK, Brocone M, Barsoum WK. 2010 Mid-America Orthopaedic Association Physician in Training Award: predictors of early adverse outcomes after knee and hip arthroplasty in geriatric patients. Clin Orthop Relat Res 2011;469(5):1391-400. Crossref

12. Perka $\mathrm{C}$, Arnold $\mathrm{U}$, Buttgereit F. Influencing factors on perioperative morbidity in knee arthroplasty. Clin Orthop Relat Res 2000;378:183-91. Crossref

13. Gill GS, Mills D, Joshi AB. Mortality following primary total knee arthroplasty. J Bone Joint Surg Am 2003;85(3):432-5. Crossref

14. Solomon DH, Chibnik LB, Losina E, Huang J, Fossel AH, Husni E, Katz JN. Development of a preliminary index that predicts adverse events after total knee replacement. Arthritis Rheum 2006;54(5):1536-42. Crossref

15. Charlson ME, Pompei P, Ales KL, MacKenzie CR. A new method of classifying prognostic comorbidity in longitudinal studies: development and validation. J Chronic Dis 1987;40(5):373-83. Crossref

16. Weaver F, Hynes D, Hopkinson W, Wixson R, Khuri S, Daley J, Henderson WG. Preoperative risks and outcomes of hip and knee arthroplasty in the Veterans Health Administration. J Arthroplasty 2003;18(6):693-708. Crossref

17. Pugely AJ, Martin CT, Gao Y, Mendoza-Lattes S, Callaghan JJ. Differences in short-term complications between spinal and general anesthesia for primary total knee arthroplasty. J Bone Joint Surg 2013;95(3):193-9. Crossref

18. Kendall SJ, Weir J, Aspinall R, Henderson D, Rosson J. Erythrocyte transfusion causes immunosuppression after total hip replacement. Clin Orthop Relat Res 2000;381:14555. Crossref

19. Pulido L, Ghanem E, Joshi A, Purtill JJ, Parvizi J. Periprosthetic joint infection: the incidence, timing, and predisposing factors. Clin Orthop Relat Res 2008;466(7):1710-5. Crossref

20. Laskin RS. Minimally invasive total knee arthroplasty: the results justify its use. Clin Orthop Relat Res 2005;440:54-9. Crossref
21. Laskin RS, Beksac B, Phongjunakorn A, Pittors K, Davis J, Shim JC, Pavlov H, Petersen M. Minimally invasive total knee replacement through a mini-midvastus incision: an outcome study. Clin Orthop Relat Res 2004;428:74-81. Crossref

22. Caillouette JT. Anzel SH. Fat embolism syndrome following the intramedullary alignment guide in total knee arthroplasty. Clin Orthop Relat Res 1990:(251):198-9. Crossref

23. Kolisek FR, Bonutti PM, Hozack WJ, Purtill J, Sharkey PF, Zelicof SB, Ragland PS, Kester M, Mont MA, Rothman $\mathrm{RH}$. Clinical experience using a minimally invasive surgical approach for total knee arthroplasty: early results of a prospective randomized study compared to a standard approach. J Arthroplasty 2007;22(1):8-13. Crossref

24. Berger RA, Sanders S, Gerlinger T, Della Valle C, Jacobs JJ, Rosenberg AG. Outpatient total knee arthroplasty with a minimally invasive technique. J Arthroplasty 2005;20:33-8. Crossref

25. Gandhi R, Smith H, Lefaivre KA, Davey JR, Mahomed NN. Complications after minimally invasive total knee arthroplasty as compared with traditional incision techniques: a metaanalysis. J Arthroplasty 2011;26(1):29-35. Crossref

26. Lovald ST, Ong KL, Malkani AL, Lau EC, Schmier JK, Kurtz SM, Manley MT. Complications, mortality, and costs for outpatient and short-stay total knee arthroplasty patients in comparison to standard-stay patients. J Arthroplasty 2014;29(3):510-5. Crossref

27. SooHoo NF, Lieberman JR, Ko CY, Zingmond DS. Factors predicting complication rates following total knee replacement. J Bone Joint Surg Am 2006;88(3):480-5. Crossref

28. Hart A, Bergeron SG, Epure L, Huk O, Zukor D, Antoniou J. Comparison of US and Canadian perioperative outcomes and hospital efficiency after total hip and knee arthroplasty. JAMA Surg 2015;150(10):990-8. Crossref

29. Parvizi J, Sullivan TA, Trousdale RT, Lewallen DG. Thirty-day mortality after total knee arthroplasty. J Bone Joint Surg Am 2001;83(8):1157-61. Crossref

30. Parry MC, Smith AJ, Blom AW. Early death following primary total knee arthroplasty. J Bone Joint Surg Am 2011;93(10):948-53. Crossref 